Artículos Científicos

\title{
Análisis de la literatura: tópicos manifiestos y tendencias de las investigaciones realizadas en jóvenes con aptitudes sobresalientes
}

\author{
Literature analysis: the manifest topics and tendency of investigations \\ made in young gifted people
}

\section{Análise da literatura: temas manifestos e tendências da pesquisa realizada em jovens com habilidades destacadas}

\author{
Alejandro Jesús Robles Ramírez \\ Universidad Autónoma de Ciudad Juárez, México \\ aroblesj93@gmail.com \\ https://orcid.org/0000-0002-4560-9501 \\ Diana Irasema Cervantes Arreola \\ Universidad Autónoma de Ciudad Juárez, México \\ diana.cervantes@uacj.mx \\ https://orcid.org/0000-0003-2353-1309
}

\section{Resumen}

En este artículo se ofrece una revisión del estado de la cuestión, así como un análisis sobre el tema de los jóvenes con aptitudes sobresalientes (AS). El objetivo central fue dar a conocer cuáles son los tópicos que se han estudiado sobre dicho tema dentro del nivel medio superior y las dificultades que esos estudiantes presentan, así como las tendencias en el tipo de trabajo de investigación que se han efectuado en relación con estos casos en diferentes países durante el periodo 2010-2019. De una búsqueda en diferentes bases de datos, se obtuvieron 33 artículos que mencionan específicamente las AS en nivel medio superior. Los resultados 


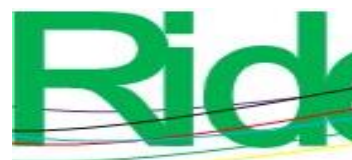

Revista Iberoamericana para la Investigación y el Desarrollo Educativo ISSN $2007-7467$

demuestran que existen escasos trabajos dedicados especialmente a jóvenes con AS en el nivel medio superior, aunque también se hallaron datos generales sobre algunos tópicos, como dificultades que presenta esa población, rasgos característicos de estos y factores familiares inmersos. En conclusión, se puede afirmar que se necesitan más investigaciones, sobre todo en México, para mejorar el apoyo, la detección y la atención de jóvenes con AS, pues las dificultades de índole psicosocial o la relación con los pares son aspectos que deben ser abordados, así como las posibilidades de atender a estos alumnos o el seguimiento que se les debe dar a sus familias, ya que esta institución funge como facilitadora de procesos pedagógicos y de apoyo.

Palabras clave: aptitud sobresaliente, características, dificultades, factores familiares, medio superior.

\section{Abstract}

This article shows the state of the art and analysis in the young gifted students theme. The objective is to show topics young gifted people in high school level and the type of research work that has been carried out in relation in this area, in different countries, during the period 2010-2019. In the search in different bases, it was obtained 33 articles bases that mention gifted in high school. This revision generates results of low work in the case of young gifted people and general data on some topics that these reviewed provide, like their difficulties in that cases, their characteristics and familiar immersed factors. It concludes that is necessary more research, especially in Mexico, to improve the support and attention to this cases, because the psychological difficulties or the relation with pairs is important to work and approach possible interventions for the gifted's families, as they are a pedagogical facilitator and process support.

Keywords: Gifted, characteristics, difficulties, familiar factors, high school. 


\section{Resumo}

Este artigo oferece uma revisão do estado da questão, bem como uma análise sobre o assunto de jovens com habilidades destacadas (EA). O objetivo principal era dar a conhecer os temas estudados sobre o assunto no nível médio superior e as dificuldades que esses alunos apresentam, bem como as tendências no tipo de trabalho de pesquisa realizado em relação a eles. casos em diferentes países durante o período 2010-2019. A partir de uma pesquisa em diferentes bases de dados, foram obtidos 33 artigos que mencionam especificamente o EA no nível médio superior. Os resultados mostram que existem poucos empregos dedicados especialmente aos jovens com EA no nível médio superior, embora também tenham sido encontrados dados gerais sobre alguns tópicos, como dificuldades apresentadas por essa população, características características desses e fatores familiares imersos. Concluindo, pode-se afirmar que são necessárias mais pesquisas, principalmente no México, para melhorar o apoio, a detecção e o atendimento de jovens com SA, uma vez que dificuldades psicossociais ou o relacionamento com colegas são aspectos que devem ser abordados. , bem como as possibilidades de atendimento a esses alunos ou o acompanhamento que deve ser dado às famílias, uma vez que essa instituição atua como facilitadora de processos pedagógicos e de apoio.

Palavras-chave: aptidão destacada, características, dificuldades, fatores familiares, meio superior.

Fecha Recepción: Mayo 2019

Fecha Aceptación: Diciembre 2019 


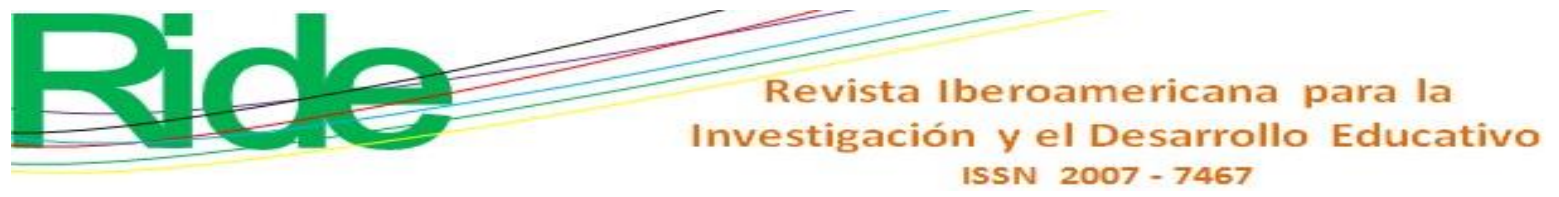

\section{Introducción}

En décadas recientes, si bien es cierto que ha tomado gran relevancia el tema sobre una adecuada educación para las personas con aptitudes sobresalientes (AS), así como sobre el beneficio que este recurso humano aporta para el desarrollo general de cada país (Kalashi, Sokrzadeh y Kamkari, 2018; Seaton, Marsh, Parker, Craven y Yeung, 2015; Valdés, Vera y Carlos, 2013), también los es que aún falta proponer actividades que les permitan a estos individuos desarrollar al máximo su proceso de enseñanza-aprendizaje. Por eso, en diversos lugares del mundo se ha comenzado a promover una intervención institucional global orientada por las distintas organizaciones educativas, entidades encargadas de potenciar y atender los aprendizajes de las personas con AS (Martín y Vargas, 2014).

Una muestra son las escuelas académicamente selectivas de Estados Unidos, las cuales se enfocan en estos casos para presentarles a los alumnos un abanico más específico de conocimientos relacionados con las ciencias en general, las tecnologías, las ingenierías y las matemáticas [STEM] (Fraleigh-Lohrfink, Schneider, Whittington y Feinberg, 2013; Pride, 2014; Young, Young y Ford, 2017). Estas iniciativas, sin embargo, son escasas en instituciones de nivel medio superior, donde solo se suelen desarrollar actividades generales, y no específicas, como sí sucede en el nivel de pregrado (Fraleigh-Lohrfink et al., 2013).

Otro de los puntos importantes de la atención de las personas con estas aptitudes es la intervención, la cual en ocasiones se lleva a cabo agrupando a los estudiantes con pares que también tienen AS, pues de esa manera se fomentan clases más desafiantes. Sin embargo, cuando este tipo de actividades no son aprovechadas, y a los estudiantes con AS se les agrupa con alumnos regulares, se ha demostrado que en los primeros aumentan los niveles en variables como aburrimiento y autoconcepto académico (Prekel, Götz y Frenzel, 2012). En otras palabras, los educandos con AS muestran mayores rangos de tedio cuando no son sobrerretados.

En lo concerniente al autoconcepto, los anteriores autores demostraron que cuando un alumnado es cambiado de un grupo regular a uno exclusivo se produce un decremento significativo del autoconcepto, aunque en el caso de los educandos con AS su autoconcepto aumenta en comparación con el de los estudiantes sin AS, pues son aceptados dentro de un grupo exclusivo, lo cual eleva su motivación. De lo anterior se puede inferir que el nivel de reto y la motivación son factores indispensables para que los estudiantes con AS puedan 
combatir el aburrimiento (Chae y Gentry, 2011).

El problema, no obstante, se encuentra en saber encontrar la intensidad óptima del estímulo-reto, pues de ese modo se evita situarse en alguno de los extremos de dicha díada, lo cual es necesario para que toda persona (sea o no sobresaliente) se pueda sentir desafiada y motivada al mismo tiempo, claves para potenciar el avance intelectual y el rendimiento académico (Valdés, Carlos, Vera y Montoya, 2012).

Sobre este tema del autoconcepto, también vale destacar el trabajo de Ordaz y Acle (2012), quienes explican que no existe diferencia en dicho criterio cuando se comparan a hombres y mujeres con alta capacidad. De hecho, según Hormazábal-Peralta, Espinoza, Cáceres y Lizana (2018), los educandos con AS suelen tener bajos niveles de sobrepeso y obesidad, pues tienden a realizar más ejercicio del pautado en los currículos escolares.

Otro elemento que se debe tomar en cuenta para atender a esta población tiene que ver con la oferta de talleres extracurriculares que fomenten el gusto por la investigación (López, 2015), lo cual se puede concretar a partir de la enseñanza de temas novedosos que potencien sus habilidades. Estos esfuerzos basados en planes extracurriculares, agrupaciones, escuelas selectivas y descubrimiento de características pueden ayudar al estudiante a elegir una futura carrera profesional, lo cual lógicamente debe estar planificado desde una adecuada tutoría o consejería, eslabón esencial para conseguir los objetivos trazados.

Para ello, el tutor o consejero debe conocer las particularidades raciales, económicas, sociales, así como los problemas de frustración o ansiedad que puedan tener las personas con AS para decidir optar por una u otra carrera (Cross y Dawn, 2010; Ordaz y Acle, 2012; Ozcan, 2017).

Por estas razones, el objetivo del presente trabajo es efectuar una revisión del estado de la cuestión sobre los tópicos y el trabajo (tipo, lugares y año) desarrollado en diferentes países para atender a los jóvenes con AS que cursan el nivel medio superior. 


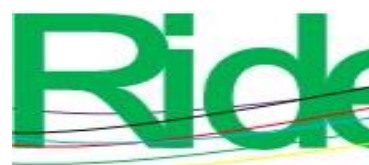

Revista Iberoamericana para la

Investigación y el Desarrollo Educativo ISSN $2007-7467$

\section{Método}

De acuerdo con Huergo-Tobar (2015), los estados de la cuestión o análisis documentales son relevantes porque propician "una base para desarrollar la lectura y la comprensión de textos, enfocados a la producción de escritos de origen científico que coadyuven en la construcción de conocimiento mediante el empleo del pensamiento crítico" (p. 1). Aunado a esto, Gómez, Galeano y Jaramillo (2015) argumentan que el estado de la cuestión intenta trascender los datos y las referencias de lo conocido hasta el momento para "construir un orden coherente que explique y totalice los significados sobre un fenómeno en particular" (p. 424), lo cual se logra organizando y estructurando los datos de investigaciones aisladas.

Esta investigación se efectuó a través de la revisión y el análisis de la literatura disponible sobre el referido tema en diversas bases de datos, como Scientific Electronic Library Online (Scielo), Red de Revistas Científicas de América Latina y el Caribe, España y Portugal (Redalyc), Dialnet y EBSCOhost.

Las palabras clave de la búsqueda inicial fueron: altas capacidades, superdotación, aptitudes sobresalientes, sobredotación, aptitudes sobresalientes en bachillerato, aptitudes sobresalientes en nivel medio superior, gifted high school students y high school gifted students. En principio se encontraron aproximadamente 9000 estudios sobre jóvenes con aptitudes sobresalientes; sin embargo, se realizó un proceso de depuración que permitió detectar 33 artículos vinculados específicamente con las aptitudes sobresalientes en el nivel medio superior. En concreto, se tomó el nombre referido a la diferenciación dentro de las palabras y concepciones de alto rendimiento o talento y las AS o sobredotación. Esto significa que se discriminaron aquellos escritos que tomaban su papel dentro del área del talento, siendo esto la primera pauta de inclusión de los escritos. Seguido de esto, únicamente se eligieron artículos de revistas científicas. Como tercer criterio de selección se tomaron aquellas investigaciones publicadas entre 2010 y 2019. Por último, no se tomaron en cuenta trabajos que exigían una subscripción monetaria a sitios de internet o a revistas especializadas ni artículos que estuvieran incompletos (p. ej., que solo tenían disponibles su abstract o resumen). 


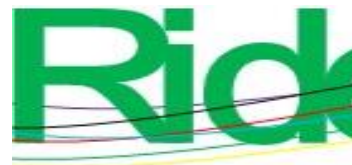

Revista Iberoamericana para la

Investigación y el Desarrollo Educativo ISSN $2007-7467$

Asimismo, vale acotar que en México se utiliza el término aptitudes sobresalientes para referirse a la sobredotación/superdotación/altas capacidades, mientras que en otros países como España y Chile se emplea las expresión altas capacidades para denotar tanto las AS como el talento. Esto obligó a realizar una lectura detallada para discriminar aquellas obras que usaban el vocablo altas capacidades cuando hacían alusión al talento. Asimismo, cabe resaltar que en el caso de la literatura de lengua inglesa, la palabra gifted es la más usual para referirse a las AS.

En cuanto al análisis, este se realizó mediante el llenado de una tabla de Excel con la información general y resumida de los documentos. En una primera lectura lineal se pudo categorizar la información y el tema de los artículos para buscar los tópicos en que se engloban. Posteriormente, y dada la similitud en cuanto a los tópicos seleccionados, se efectuó una segunda lectura de tipo transversal que permitió generar un cotejo paralelo para detectar las similitudes, vacíos, complementariedades y para corroborar, ampliar y encontrar contrariedades entre las referencias. Hecha esta observación, emergieron los tópicos que se presentan en el siguiente apartado.

\section{Resultados}

En primera instancia, se comenzará con la introducción del análisis bibliográfico de los artículos revisados para indicar cuáles países [contextualizar] están tomando en cuenta la importancia de realizar estudios para conocer las fortalezas, carencias, debilidades y características de la población con AS en el nivel medio superior. De este modo se podrán introducir los temas abarcados en los escritos.

En el otro de los puntos establecidos en el objetivo (tendencias del tipo de trabajo investigativo, lugares y años de los estudios realizados sobre los jóvenes con AS de nivel medio superior), se encontró que las publicaciones abarcaban los continentes americano (México, Estados Unidos, Canadá y Chile), europeo (España, Francia, Portugal, Austria, Rumania y Turquía) y asiático (Irán, Corea del Sur, Israel, Baréin y China). Se debe aclarar que uno de los escritos fue publicado por autores de dos países (Corea del Sur y Estados Unidos), razón la cual en la tabla 1 aparecen 34 documentos (Ver tabla 1). 
Tabla 1. Número de publicaciones encontradas por país

\begin{tabular}{|c|c|}
\hline $\begin{array}{c}\text { País de } \\
\text { origen }\end{array}$ & $\begin{array}{c}\mathbf{N}^{\circ} \text { de } \\
\text { publicaciones }\end{array}$ \\
\hline Estados Unidos & 8 \\
\hline México & 4 \\
\hline España & 4 \\
\hline Chile & 3 \\
\hline Turquía & 2 \\
\hline Irán & 2 \\
\hline Israel & 2 \\
\hline Canadá & 2 \\
\hline Francia & 1 \\
\hline Portugal & 1 \\
\hline Corea del Sur & 1 \\
\hline Austria & 1 \\
\hline Rumania & 1 \\
\hline China & 1 \\
\hline Bahréin & 1 \\
\hline Funte: & \\
\hline
\end{tabular}

Fuente: Elaboración propia

Por otra parte, en la figura 1 se puede percibir una variación en el número de publicaciones según cada año. En síntesis, son visibles los picos en los años 2011, 2013 y 2017, aunque también los vacíos en 2016 y 2019.

Figura 1. Publicaciones por año (periodo 2010-2019)

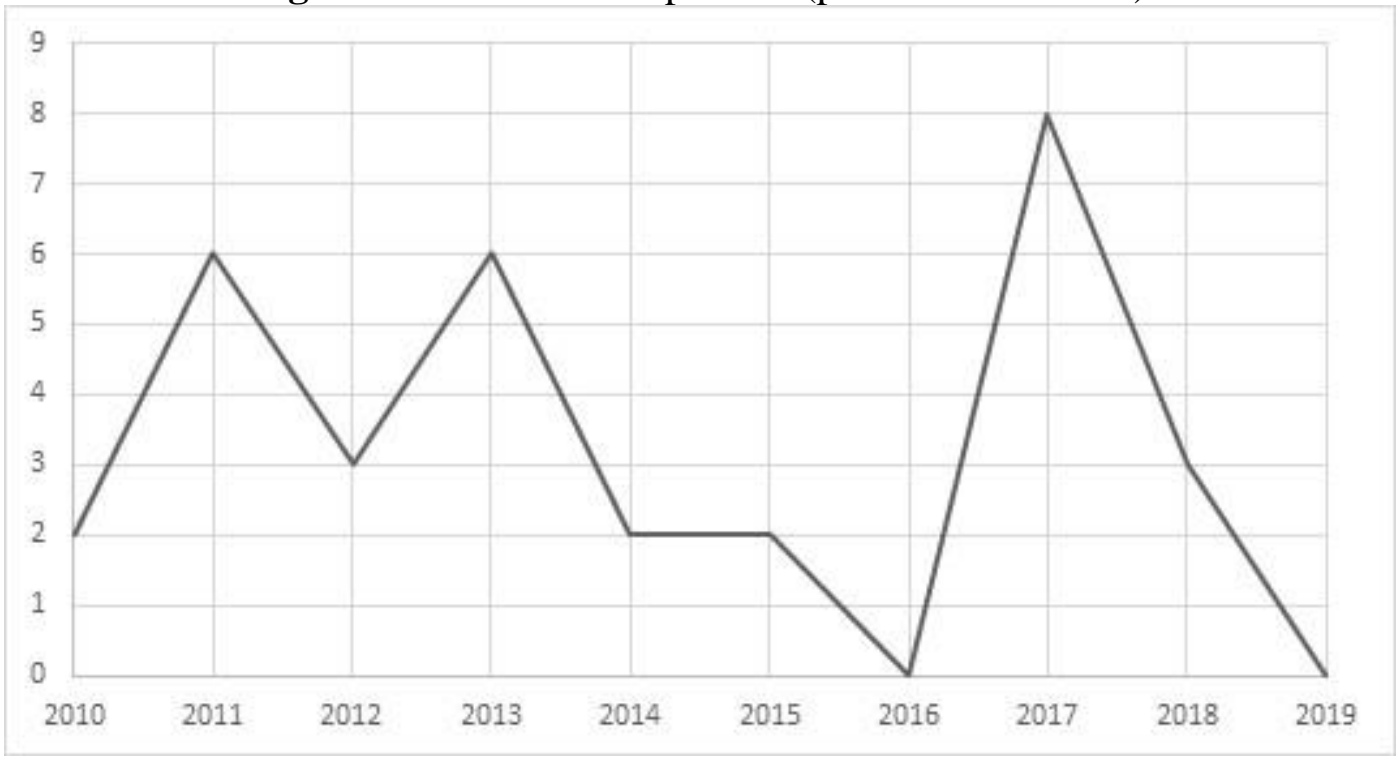

Fuente: Elaboración propia 


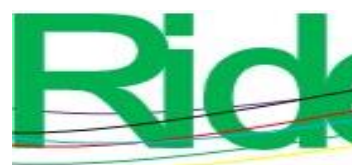

Revista Iberoamericana para la Investigación y el Desarrollo Educativo ISSN $2007-7467$

En cuanto al nivel específico de los trabajos realizados, en 22 se abordan poblaciones de bachillerato, mientras que en 11 artículos los autores se enfocan en estudiantes tanto de nivel básico como medio superior. En concreto, $33 \%$ de las publicaciones se centran en el nivel básico (preescolar, primaria y secundaria), mientras que $67 \%$ se dedica exclusivamente al nivel medio superior.

Sobre la naturaleza de las investigaciones, 5 son cualitativos, con diseños como el estudio de caso, la estrategia documental retróspectiva, la metodología narrativa y las historias de vida (tabla 2), mientras que 25 son cuantitativos, de tipo comparativos —2 descriptivos-correlacionales y 2 longitudinales (tabla 3) - También cabe indicar que algunas investigaciones emplean más de un tipo de diseño, de ahí que el total sea superior al número general de estudios hallados; además, en 3 trabajos no se especifica la naturaleza de la indagación o eran revisiones de literatura.

Tabla 2. División y número de publicaciones cualitativas conforme a su diseño o tipo

\begin{tabular}{|c|c|c|c|c|}
\hline $\begin{array}{c}\text { Método de } \\
\text { la } \\
\text { publicación } \\
\text { (cualitativo) }\end{array}$ & $\begin{array}{c}\text { Estudio } \\
\text { de caso }\end{array}$ & $\begin{array}{c}\text { Estrategia } \\
\text { documental } \\
\text { de carácter } \\
\text { retrospectivo }\end{array}$ & $\begin{array}{c}\text { Sin } \\
\text { especificar }\end{array}$ & $\begin{array}{c}\text { Historia } \\
\text { de vida }\end{array}$ \\
\hline $\begin{array}{c}\text { Número de } \\
\text { publicaciones }\end{array}$ & 1 & 1 & 1 & 1 \\
\hline
\end{tabular}

Fuente: Elaboración propia

Tabla 3. División y número de publicaciones cuantitativas conforme a su diseño o tipo

\begin{tabular}{|c|c|c|c|}
\hline $\begin{array}{c}\text { Método de la } \\
\text { publicación } \\
\text { (cuantitativo) }\end{array}$ & $\begin{array}{c}\text { Estudio } \\
\text { comparativo }\end{array}$ & $\begin{array}{c}\text { Descriptivo- } \\
\text { correlacional }\end{array}$ & Longitudinal \\
\hline $\begin{array}{c}\text { Número de } \\
\text { publicaciones }\end{array}$ & 20 & 9 & 3 \\
\hline
\end{tabular}

Fuente: Elaboración propia

Ahora bien, si se dividen los artículos analizados según el idioma en que fueron escritos, $67 \%$ están presentados en inglés, $12 \%$ en portugués y $21 \%$ en español. Esto demuestra que existe una mayor tendencia a publicar en lengua anglosajona, incluso en países donde dicha lengua no es la oficial (Ver tabla 4). 


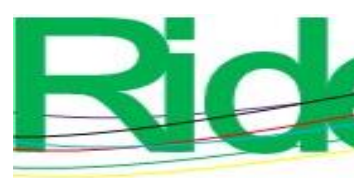

Revista Iberoamericana para la

Investigación y el Desarrollo Educativo

ISSN $2007-7467$

Tabla 4. Número de publicaciones conforme al idioma que fueron escritos

\begin{tabular}{|c|c|c|c|}
\hline $\begin{array}{c}\text { Idioma de la } \\
\text { publicación }\end{array}$ & Inglés & Español & Portugués \\
\hline $\begin{array}{c}\text { Número de } \\
\text { publicaciones }\end{array}$ & 22 & 10 & 1 \\
\hline
\end{tabular}

Fuente: Elaboración propia

Asimismo, y en cuanto a los tópicos que se abordan para el caso de los jóvenes con AS de nivel medio superior, se puede decir que la mayoría se enfoca en la dificultad que presentan esos educandos a la hora de que sus necesidades educativas sean satisfechas. Igualmente, se intenta hacer un esfuerzo por detectar los rasgos que caracterizan a las personas con AS, así como sus principales dificultades vinculadas con el entorno o situación familiar. En este sentido, a continuación se exponen los tópicos que se encontraron en las investigaciones revisadas:

\section{Tópico 1: Las dificultades que presentan los jóvenes con AS}

A pesar de los esfuerzos realizados, aún existe dificultad por parte de las escuelas para crear planes que permitan el enriquecimiento de los estudiantes con AS (Villatte, Courtinat-Camps y Léonardis, 2014; Valdés, Sánchez y Yañez, 2013; Valdés et al., 2013). De hecho, en cuanto a las escuelas selectivas, no todos los educandos poseen las mismas posibilidades de entrar a dichas instancias (Fraleigh-Lohrfink et al., 2013; Young et al., 2017). En tal sentido, en algunos centros se percibe una tendencia discriminatoria según la raza (se incluyen a las personas de color blanco o asiáticos, y se excluyen a las razas afroamericanas, latinas e indias) (Fraleigh-Lohrfink et al., 2013; Pride, 2014; Young et al., 2017).

Por otra parte, Jiménez y García (2013) explican que dentro de España las instituciones clasificadas por excelencia (STEM) captan principalmente a los mejores estudiantes. Sin embargo, vale destacar que las diferencias que pueden presentar los educandos de las residencias STEM suelen generar necesidades y afectaciones de índole psicosocial, como desenvolvimiento con dificultad en la escuela, problemas en la elección de carrera y baja autoestima, así como dificultades vinculadas con la pubertad, las relaciones de pareja, la creación de nuevas amistades y la formación de identidad. Esto se agrava debido a que estos estudiantes no encuentran apoyo en sus amigos ni familiares. De hecho, según 


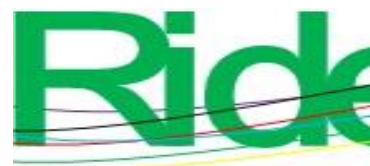

Revista Iberoamericana para la Investigación y el Desarrollo Educativo ISSN 2007 - 7467

diversos autores, esto se genera porque los dotados poseen un nivel menor de habilidad mental para tolerar los problemas (Cross y Dawn, 2010; Kalashi et al., 2017).

Los anteriores resultados, sin embargo, son distintos a los reportados por Özbey, Sarıçamy y Karduz (2018), quienes han encontrado que los estudiantes con AS tienen menos probabilidades de sentirse desequilibrados y extraños en escuelas ajenas del lugar de origen y tienen menos dificultades para hacer amigos que sus compañeros regulares que viven en sus propios países o lugares de origen.

Aunado a esto, Lupu (2012), Al-Hamdan, Al-Jasim y Abdulla (2017) y Özbey et al. (2018) encontraron en sus estudios que los adolescentes con AS poseen una inteligencia emocional, una empatía y un sentido de comunidad mayor que las personas sin AS. Igualmente, Chen, Cheung, Fan y Wu (2017) mencionan que los educandos con AS poseen una resiliencia alta; incluso cuando estos estudiantes se trazaban una meta, se esforzaban por superar las dificultades presentadas, lo cual demuestra que el logro de los objetivos de carrera y la capacidad de recuperación académica están relacionados. A pesar de esto, se debe resaltar que estas personas necesitan una guía o consejería debido a que pueden extrañar su casa o manifestar tendencias suicidas (Cross y Dawn, 2010).

Otro aspecto a tomar en cuenta para estos alumnos tiene que ver con el acoso escolar, aunque Según Ryo, Wang, Swearer y Park (2017) reportan que no existen diferencias en cuanto a ser víctimas de bullying entre estudiantes con AS y sus pares no dotados. Estos autores, de hecho, mencionan que los educandos con AS que están en los grados intermedios tienen más probabilidades de ser propiciadores del acoso escolar, lo cual suele disminuir cuando se encuentran en grados superiores. Según estos autores, esta posibilidad de participar en agresiones aumenta cuando ellos han tenido experiencias sociales negativas de intimidación, lo cual se trasforma más adelante en sentimientos de ira.

En cuanto a algunos mitos que recaen sobre estos estudiantes, se debe combatir la idea de que estas personas no necesitan ningún tipo de ayuda pedagógica, pues se suele creer que ellos pos sí mismo pueden salir adelante. Por ello, no se debe pensar que las AS son garantía absoluta de éxito y alto rendimiento académico (Martín y Vargas, 2014). Al respecto, Valdés et al. (2012) afirman que "los estudiantes sobresalientes necesitan adaptaciones, tanto del currículo como del entorno familiar, además de profesores y padres 


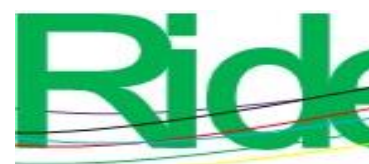

Revista Iberoamericana para la Investigación y el Desarrollo Educativo ISSN 2007 - 7467

que conozcan y puedan responder de manera efectiva a sus características y necesidades educativas" (p. 41).

En relación con las dificultades para la atención de las AS, pero desde otros agentes involucrados en los procesos pedagógicos de estos estudiantes, Conejeros-Solar, GómezArízaga y Donoso-Osorio (2013) presentan los resultados sobre la percepción de los educandos sobre la actitud de sus docentes. Según sus hallazgos, los educandos ven de manera positiva e importante a un profesor cercano que se preocupe por lo que ocurre con ellos dentro del salón de clases. Incluso valoran que los docentes presenten actividades novedosas donde se pueda aplicar la teoría aprendida.

Estas apreciaciones coindicen con lo descubierto por Chae y Gentry (2011), quienes explican que los estudiantes prefieren cátedras donde puedan percibir la conexión entre el mundo real y lo visto en clase. Además, los educandos ven muy bien al profesorado que logra formar una serie de reflexiones profundas con los temas vistos y les generan retos mentales (Conejeros-Solar et al., 2013).

Por su parte, Eilam y Vidergor (2011) realizaron un estudio con estudiantes árabes y judíos con sobredotación-AS para determinar cuáles eran las diferencias entre las percepciones de ellos sobre sus docentes en programas especiales de enriquecimiento. Los autores encontraron que los profesores deben poseer una conciencia plena de los aspectos culturales de los alumnos, ya que este criterio influye de manera significativa en las apreciaciones de sus estudiantes. Esto se debe a que los alumnos árabes, al ser más cohibidos, exteriorizan una postura de menor sensibilidad con sus docentes, a diferencia los judíos, quienes suelen ser más sentimentales con los maestros. Este factor cultural también se demuestra en la preferencia de ambas etnias en cuanto al paradigma pedagógico que orienta las actividades del docente, pues mientras que los árabes se sienten más cómodos con un profesor conductista — debido a las marcadas influencias sociales de nunca irrespetar ni cuestionar a sus maestros-, los judíos prefieren a un docente que promueva la resolución de problemas, la creatividad, el trabajo en grupo y los proyectos individuales.

Por otra parte, se debe destacar que una dificultad hallada en el presente estudio tiene que ver, en general, con el grupo de alumnos con los cuales se desarrollan las investigaciones, es decir, predominantemente personas de escuelas de educación básica, y no poblaciones del nivel medio superior. Sobre este aspecto, se debe tener en cuenta que los métodos empleados 


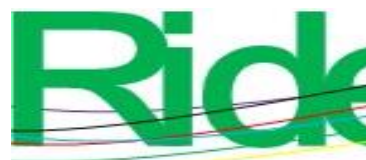

Revista Iberoamericana para la

Investigación y el Desarrollo Educativo

ISSN $2007-7467$

para el diagnóstico de las AS en educación básica resultan insuficientes para ser usados en el nivel de bachillerato, de ahí que se deban usar instrumentos enfocados en esta población, dado que existen distintos niveles y subtipos de sobredotación, incluso dentro de los talentos específicos (Leikin, Leikin, Patz-Baruch, Waisman y Lev, 2017; Ordaz y Acle, 2012; Valdés et al., 2013).

En México, este punto resulta vital porque la detección, los planes de enriquecimiento y la intervención únicamente han sido elaborados para la población de educación primaria, lo cual genera una desatención a los demás alumnos (Ordaz y Acle, 2012; Valdés et al., 2012; Valdés et al., 2013). A partir de esta afirmación, se puede deducir que resulta relevante el trabajo de adecuación curricular y la labor docente para un mayor aprendizaje y aprovechamiento de los estudiantes con AS.

\section{Tópico 2: Características encontradas en los jóvenes con AS a partir de los estudios realizados}

Las características que recientemente han sido abordadas en los estudios son el perfeccionismo y el liderazgo, en las cuales se menciona que generan dificultades de relación con los pares en el caso de ciertas personas con AS. Esto se deriva por la característica del perfeccionismo, que desencadena desacuerdos sobre cómo ver las cosas o realizarlas, pues se puede percibir que las personas con AS muestran una tendencia por el perfeccionismo al optar por una posición de liderazgo que puede estar arraigada e influenciada desde el entorno familiar. Esto significa que cuando los padres son perfeccionistas, existen mayores posibilidades de que los hijos desarrollen esas cualidades, las cuales suelen ser particularmente perjudiciales en la etapa de la adolescencia para las personas con AS, periodo de la vida donde es más habitual que se pueda generar el síndrome de burnout (Fernandes, Vasconcelos-Rapos, Bertelli y Almeida, 2011; González, 2013; González, Gómez-Arízaga y Conejeros-Solar, 2017).

Igualmente, vale comentar un estudio de French, Walker y Shore (2011), quienes demostraron que los estudiantes con AS prefieren trabajar solos cuando no sienten que sus trabajos están siendo apreciados o bien valorados. De hecho, a medida que pasa el tiempo, estos alumnos prefieren trabajar en su propio aprendizaje y logran discernir entre lo que a otros no les llama la atención y lo que a ellos sí. 


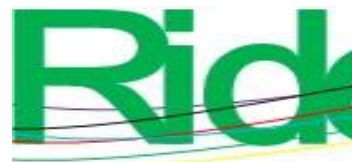

Revista Iberoamericana para la Investigación y el Desarrollo Educativo ISSN 2007 - 7467

Sobre el tema de las preferencias individuales, se destaca la investigación de Betts y McMasters (2011), quienes intentaron mostrar a tres estudiantes con sobredotación y alto rendimiento en las matemáticas una idea distinta a la enseñada en el currículo, ello mediante un programa de enriquecimiento de orden filosófico que mostraba la naturaleza de las matemáticas más desordenadas. Los resultados demuestran que los educandos sienten un total rechazo ante esta postura, con lo que los autores alegan que es un problema curricular, dado que no se presentan componentes críticos del proceso, aunque en realidad fue la decisión de la postura epistémica que eligieron los educandos (Betts et al., 2011).

Otros elementos que se deben atender son las perspectivas y preferencias de esos alumnos para intentar satisfacerlas en las actividades propuestas en clase (Chae y Gentry, 2011). Esto se debe considerar porque los estudiantes con AS suelen aprender de forma más rápida o tienen una capacidad más efectiva para conservar grandes cantidades de conocimiento, así como mayor desarrollo de experticia en el área de estudio, memoria de trabajo eficiente, pensamiento flexible, competencia lingüística y habilidad verbal, pensamiento abstracto y más capacidad metacognitiva para resolver problemas, formar relaciones inusuales entre disciplinas u objetos, realizar con éxito autoinvestigación, transferir conocimiento y aplicarlo en situaciones nuevas (Eilam y Vidergor, 2011; Snyder, Nietfeld y Linnenbrink-García, 2011). Todos estos elementos, en consecuencia, se deben aprovechar al momento de hacer adecuaciones curriculares y adaptaciones del trabajo académico.

\section{Tópico 3: Factores familiares inmersos en los casos con AS}

Los factores familiares son aspectos que, de acuerdo con Valdés et al. (2012), también se deben considerar en la historia personal de las personas con AS, pues estos influyen de diversa manera, por ejemplo, en cuanto a la generación de un entorno donde a los educandos se les facilite su crecimiento intelectual y social, y donde se les ayude a la resolución de propuestas en la toma de decisiones. En palabras de los citados autores, "la influencia de la familia se puede dar tanto a través de factores de insumo o estructurales (condición socioeconómica, nivel de escolaridad de los padres y recursos para el estudio [...]), o bien, mediante factores procesales" (p. 41). Dicho de otro modo, la familia es un elemento esencial para facilitar el proceso pedagógico de los educandos con AS, de ahí que Valdés et al. (2012) 


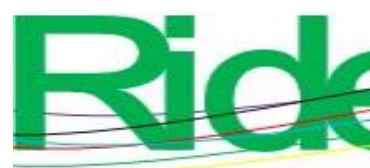

Revista Iberoamericana para la

Investigación y el Desarrollo Educativo

ISSN $2007-7467$

hayan intentado en su trabajo estandarizar un instrumento sobre el clima familiar de educandos con AS para orientar a las familias sobre la manera en que pueden ayudar a sus hijos.

Por último, se debe prever que a las personas con AS también les suele afectar ser catalogadas por sus pares no sobresalientes como individuos superdotados, lo cual puede provocar que oculten sus capacidades para ser aceptados por los demás (Pride, 2014). Esta actitud de encubrimiento se produce principalmente en la pubertad, pues los adolescentes tienden a estar más atentos ante cualquier característica distintiva de sus coetáneos (Fernandes et al., 2011).

\section{Discusión}

En cuanto a los tópicos manifiestos para estos casos, se lograron encontrar algunas de las dificultades que los jóvenes con AS presentan, como ajuste psicosocial y académico, importancia del entorno familiar (interacción, perfeccionismo, ayuda en el proceso de desarrollo), características relacionadas con su proceso de atención e intervención y elementos emocionales.

En lo referente al tipo de estudio realizado, se puede apreciar la tendencia de los escritos cuantitativos a comparar las variables entre educandos que presentan AS y quienes no tienen esta cualidad, así como entre alumnos de distintas nacionalidades. Asimismo, se suelen tomar en cuenta variables como el autoconcepto y su desarrollo en las escuelas especializadas, la motivación para combatir el aburrimiento, el acoso escolar, la composición corporal, la inteligencia emocional, la resiliencia, las percepciones del educando sobre el comportamiento de sus docentes según las variables culturales y la preferencia o no por trabajar solos, así como la elección de una futura carrera (French et al., 2011; Lupu, 2012; Ryo et al., 2017).

Igualmente, se rescatan las variables intelectuales y psicosociales que discriminan a los educandos con AS de quienes no tienen estas habilidades, así como los distintos tipos y niveles de AS en áreas específicas (como las matemáticas) y los factores psicosociales que pueden influenciar la trayectoria académica del educando (Cross y Dawn, 2010; Valdés et al., 2012; Valdés et al., 2013). Además, se pudo constatar que existe una fuerte conexión entre el perfeccionismo parental y el desarrollado por los hijos con AS (Kakavand, Kalantari, 


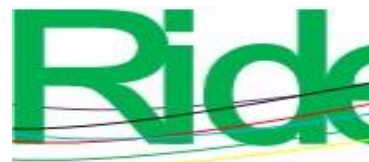

Revista Iberoamericana para la

Investigación y el Desarrollo Educativo

ISSN $2007-7467$

Noohi y Taran, 2017); esto permite inferir que las variables psicosociales, posiblemente, tengan una influencia sobre el desarrollo académico del estudiante.

Las investigaciones cualitativas, en cambio, se enfocan en la historia de vida de los alumnos con AS (Pride, 2014) o en el querer inculcar en los educandos una visión epistemológica distinta de las matemáticas mediante un enfoque narrativo (Betts et al., 2011). En otra investigación de esta naturaleza, el estudio de caso encontró resultados similares tomando en consideración el perfeccionismo a partir de los padres (González et al., 2017). Además, se logró apuntalar las necesidades de los educandos con AS según el perfil idóneo de sus docentes (Conejeros-Solar et al., 2011).

En síntesis, se puede decir que los estudios cuantitativos son los mayormente abordados en la revisión de los tópicos para los casos de AS, los cuales también promueven diversos análisis descriptivos, longitudinales y comparativos para destacar las características y dificultades de esta población.

En cuanto a los países con mayores publicaciones sobre el tema en cuestión, se puede afirmar que los más elevados porcentajes provienen de Estados Unidos y Europa, mientras que en México se pudieron encontrar cuatro estudios, un número aceptable si se compara con los de otras naciones latinoamericanas.

Finalmente, se debe indicar que 2011 y 2013 fueron los años en los cuales se halló la mayor cantidad de publicaciones, aunque vale acotar que no todos los artículos se enfocan de manera específica en las AS de nivel medio superior, sino también en otros niveles educativos.

\section{Conclusiones}

Esta investigación fortalece el conocimiento sobre el tema de las AS, pues, según el análisis efectuado, se puede afirmar no solo que existe una clara desarticulación de los escritos hallados, sino también que las características de los educandos con AS son muy dispares. Asimismo, se puede apreciar en varios estudios que la mayoría de los trabajos enuncian variables como el perfeccionismo, el autoconcepto y algunas necesidades educativas que los alumnos con AS pueden tener, así como aspectos de tipo intelectual y diversas habilidades. Sin embargo, cabe resaltar que la mayoría de trabajos sobre las AS se han centrado en etapas de la niñez, y no tanto en jóvenes de nivel medio superior o nivel 
superior, razón por la que se requieren más trabajos enfocados en esta población para ampliar el debate en torno a las posibles maneras de atender sus necesidades. En tal sentido, es erróneo pensar que estos alumnos carecen de los problemas que pueden tener las demás personas. Por ello, se debe recalcar que las personas con AS no necesariamente se adaptan de manera sencilla al medio escolarizado, pues en cuanto a la capacidad para socializar suelen tener las mismas limitaciones que sus pares no dotados.

Por otra parte, de los 33 artículos hallados sobre el tema de estudio, se puede indicar que en México se encuentra $12 \%$ del total de publicaciones (es decir, cuatro artículos), lo que sirve para ubicar al país, junto con España, en el segundo lugar (después de Estados Unidos, nación que tiene $24 \%$ de los trabajos encontrados). Aun así, hace falta publicar más trabajos en español, ya que la mayoría se divulga en lengua inglesa.

Otro punto sobresaliente es que la mayoría de los trabajos (94\%) presenta una investigación básica que solo intenta buscar relaciones entre algunos factores para fomentar el entendimiento de la realidad educativa, así como las vivencias del estudiante y sus principales características. En otras palabras, solo $6 \%$ de las indagaciones ofrece algún tipo de intervención.

En cuanto a las dimensiones epistemológicas de la sobredotación, es justo mencionar que se están comenzando a abrir caminos hacia otros tipos de AS, con lo cual se pudieran estar ampliando las concepciones en torno a este fenómeno.

Para futuros trabajos se recomienda profundizar en poblaciones de adolescentes y jóvenes con AS, en especial en lo relacionado con la diversidad de sus características, sus dificultades y sus estilos de aprendizaje, pues de ese modo se podrán hallar nuevos nichos de trabajo. En este sentido, se ha detectado que hay un número más amplio de indagaciones en el campo de las necesidades educativa especiales (NEE), especialmente de casos de discapacidad, pero se ha descuidado el terreno de las AS, sobre todo en lo referente a su proceso de detección y el seguimiento de las personas con dichas capacidades. Incluso no se ha trabajado en la adecuación del currículo para que el proceso de enseñanza-aprendizaje mejore y cubra los requerimientos de estos estudiantes.

Finalmente, se podría considerar la posibilidad de desarrollar indagaciones que incluyan no solo los casos de AS intelectuales, sino también de las facultades psicomotoras, creativas, artísticas y social-afectivas. Asimismo, se debe reflexionar sobre los recursos 


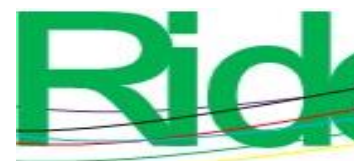

humanos que trabajarán con este alumnado, así como sobre las adaptaciones psicosocioculturales presentes en el nivel medio superior, específicamente, de México.

\section{Referencias}

Al-Hamdan, N., Al-Jasim, F. and Abdulla, A. (2017). Assessing the Emotional Intelligence of Gifted and Talented Adolescent Students in the Kingdom of Bahrain. Roeper Review, 39(2), 132-142. doi 10.1080/02783193.2017.1289462

Betts, P. and McMasters, L. (2011). Disrupting gifted teenager's mathematical identify with epistemological messiness. The Montana Mathematics Enthusiast, 8(1), 328-354. Retrieved

from https://pdfs.semanticscholar.org/7e08/10a44fd5d69430100c05a383ebce245ee33e.p df.

Chae, Y. and Gentry, M. (2011). Gifted and general high schools student's perceptions of learning and motivational constructs in Korea and United States. High Ability Studies, 22(1), 103-118. Recuperado de https://www.tandfonline.com/doi/abs/10.1080/13598139.2011.577275.

Chen, X., Cheung, H., Fan, X. and Wu, J. (2017). Factors related to resilience of academically gifted students in the chinese cultural and educational environment. Psychology in the Schools, 55(2), 107-119. Doi: https://doi.org/10.1002/pits.22044

Conejeros-Solar, M., Gómez-Arízaga, M., y Donoso-Osorio, E. (2013). Perfil docente para alumnos/as con altas capacidades. Magis: Revista Internacional de Investigación en Educación, $\quad 5(11), \quad 393-411 . \quad$ Recuperado de http://www.redalyc.org/pdf/2810/281028437007.pdf.

Cross, T. and Dawn, F. (2010). Guiding the psychosocial development of gifted students attending specialized residential SETM schools. Roeper Review, 32(1), 32-41. Retrieved from https://www.tandfonline.com/doi/abs/10.1080/02783190903386868.

Eilam, B. and Vidergor, H. (2011). Gifted Israeli student's perceptions of teacher's desired characteristics: a case of cultural orientation. Roeper Review, 33(2), 86-96. Retrieved from https://www.tandfonline.com/doi/abs/10.1080/02783193.2011.554156. 


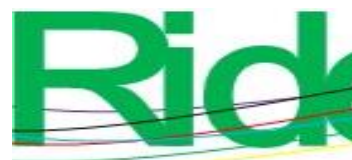

Revista Iberoamericana para la Investigación y el Desarrollo Educativo ISSN 2007 - 7467

Fernandes, H., Vasconcelos-Raposo, J., Bertelli, R. e Almeida, L. (2011). Satisfação escolar e bem-estar psicológico em adolescentes portugueses. Revista Lusófona de Educação, 18(8), 155-172. Recuperado de https://www.researchgate.net/publication/260512401_Satisfacao_escolar_e_bemestar_psicologico_em_adolescentes_portugueses.

Fraleigh-Lohrfink, K., Schneider, M., Whittington, D. and Feinberg, A. (2013). Increase in science research commitment in a didactic and laboratory-based program targeted to gifted minority high- school students. Roeper Review, 35(1), 18-26. Doi: 10.1080/02783193.2013.740599

French, L., Walker, C. and Shore, B. (2011). Facilitating student's work. Do gifted students really prefer to work alone? Roeper Review, 33(3), 145-159. Doi: 10.1080/02783193.2011.580497

Gómez, M., Galeano, C. y Jaramillo, D. A. (2015). El estado del arte: una metodología de investigación. Revista Colombiana de Ciencias Sociales, 6(2), 423-442. Recuperado de

http://bibliotecadigital.udea.edu.co/bitstream/10495/6843/1/G\%C3\%B3mezMaricell y_2015_EstadoArteMetodolog\%C3\%ADa.pdf.

González, A. (2013). El alumnado con altas capacidades: liderazgo, educación inclusiva y talento. Revista de Ciencias de la Información, 29(29), 1-26. Recuperado de https://dialnet.unirioja.es/servlet/articulo?codigo $=5629789$.

González, A., Gómez-Arízaga, M. y Conejeros-Solar, M. (2017). Caracterización del perfeccionismo en estudiantes con alta capacidad: un estudio de casos exploratorios. Revista de Psicología, 35(2), 581-616. Recuperado de http://revistas.pucp.edu.pe/index.php/psicologia/article/view/18796/19017.

Hormazábal-Peralta, A., Espinoza, J., Cáceres, P. y Lizana, P. (2018). Adolescents with high intellectual ability: differences in body composition and physical activity by sex. Nutrición Hospitalaria, 35(1), 38-43. Recuperado de https://www.ncbi.nlm.nih.gov/pubmed/29565147.

Huergo-Tobar, P. (2015). Importancia y pasos para la elaboración del estado del arte en un anteproyecto o proyecto de investigación. Bogotá: Ediciones Universidad Cooperativa de Colombia. Doi: http://dx.doi.org/10.16925/greylit.1073 


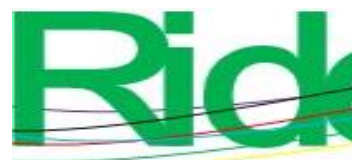

Revista Iberoamericana para la Investigación y el Desarrollo Educativo ISSN 2007 - 7467

Jiménez, C. y García, R. (2013). Los alumnos más capaces en España. Normativa e incidencia en el diagnóstico y la educación. Revista Española de Orientación y Psicopedagogía, 24(1), 7-4. Recuperado de http://revistas.uned.es/index.php/reop/article/view/11267/pdf.

Kakavand, A., Kalantari, S., Noohi, S., y Taran, H. (2017). Identifying the relationship of parenting styles and parent's perfectionism with normal student's and gifted student's perfectionism. Independent Journal of Management \& Production, 8(1), 108-123. Retrieved fromhttp://www.ijmp.jor.br/index.php/ijmp/article/view/501/620.

Kalashi, Z., Sokrzadeh, S. and Kamkari, K. (2018). Investigating The Personality Traits of Gifted Adolescents. Revista Românească pentru Educaţie Multidimensională, 10(1), 47-63. Doi: 10.18662/rrem/36

Leikin, R., Leikin, M., Patz-Baruch, N., Waisman, L. and Lev, M. (2017). On the four types of characteristics of super mathematically gifted students. High Ability Studies, 28(1), 107-125. Doi: doi.org/10.1080/13598139.2017.1305330

López, L. (2015). Taller práctico: desenmascarando los medios. Aularia: Revista Digital de Comunicación, $4(1)$, 17-76. Recuperado

de https://dialnet.unirioja.es/servlet/articulo?codigo=5096215.

Lupu, V. (2012). Emotional Intelligence in Gifted and Non-Gifted High School Students. Buletin Stiintific, 17(2), 128-132. Retrieved from http://ezproxy.uacj.mx/login?url=http://search.ebscohost.com/login.aspx?direct=tru $\mathrm{e} \& \mathrm{db}=\mathrm{a} 9 \mathrm{~h} \& \mathrm{AN}=84432110 \&$ lang=es\&site=eds-live.

Martín, R. y Vargas, M. (2014). Altas capacidades en la escuela inclusiva. Revista Padres y Maestros, 358, 39-43. Recuperado de https://revistas.comillas.edu/index.php/padresymaestros/article/view/4087/5164.

Ordaz, G. y Acle, G. (2012). Perfil psicosocial de adolescentes con aptitudes sobresalientes de un bachillerato público. Electronic Journal of Research in Educational Psychology, 10(3), 1267-1298. Recuperado de http://ojs.ual.es/ojs/index.php/EJREP/article/view/1534.

Özbey, A., Sarıçamy, H. and Karduz, F. (2018). The examination of emotional intelligence, sense of community, perception of social values in gifted and talented students. Journal of Educational Sciences y Psychology, 8(70), 64-79. Retrieved from 
https://www.researchgate.net/publication/329626546_The_examination_of_emotio nal_intelligence_sense_of_community_perception_of_social_values_in_gifted_and _talented_students_Ustun_zekali_ve_yetenekli_ogrencilerde_duygusal_zeka_toplul uk_hissi_ve_toplumsa.

Ozcan, D. (2017). Career decision-making of the gifted and talented. South African Journal of Education, 37(4), 1-8. Retrieved from http://www.scielo.org.za/scielo.php?script=sci_arttext\&pid=S025601002017000400007.

Prekel, F., Götz, T. and Frenzel, A. (2012). Ability of grouping of gifted students: effects on academic self-concept and borebom. British Journal of Educational Psychology, 80(3), 451-472. Recuperado de https://pdfs.semanticscholar.org/ec0a/6d0ab653df5c167e1a39d9e76551c7ba206c.pd f.

Pride, L. (2014). Using learning stories to capture "Gifted" and "Hard worker" mindsets within a NYC specialized high school for the sciences. Theory Into Practice, 53(1), 41-47. Doi: 10.1080/00405841.2014.862121

Ryo, J., Wang, C., Swearer, S. and Park, S. (2017). Investigation of Transitions in Bullying/Victimization Statuses of Gifted and General Education Students. Exceptional Children, 83(4), 396-411. Doi: https://doi.org/10.1177/0014402917698500

Seaton, M., Marsh, H., Parker, P., Craven, R. and Yeung, A. (2015). The reciprocal effects model revisited: extending its reach to gifted students attending academically selective schools. Gifted Child Quarterly, 59(3), 143-156. Retrieved from http://citeseerx.ist.psu.edu/viewdoc/download?doi=10.1.1.830.170\&rep=rep1\&type $=$ pdf.

Snyder, K., Nietfeld, J. and Linnenbrink-García, L. (2011). Giftedness and metacognition: a short-term longitudinal investigation of metacognitive monitoring in the classroom. Gifted Child Quarterly, 53(3), 181-193. Doi: 10.1177/0016986211412769

Valdés, A., Carlos, E., Vera, J. y Montoya, G. (2012). Propiedades psicométricas de un instrumento para medir las relaciones familiares en adolescentes intelectualmente 


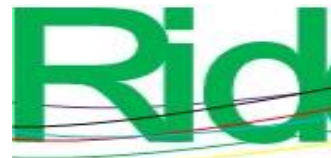

Revista Iberoamericana para la Investigación y el Desarrollo Educativo ISSN $2007-7467$

sobresalientes. Pensamiento Psicológico, 10(1), 39-50. Recuperado de http://www.redalyc.org/articulo.oa?id=80124028003.

Valdés, A., Sánchez, P. y Yáñez, A. (2013). Perfiles de estudiantes mexicanos con aptitudes intelectuales sobresalientes. Acta Colombiana de Psicología, 6(1), 25-33. Recuperado de http://www.redalyc.org/articulo.oa?id=79829185003.

Valdés, A., Vera, J. y Carlos, E. (2013). Variables que diferencian a estudiantes de bachillerato con y sin aptitudes sobresalientes. Revista Electrónica de Investigación Educativa, $\quad$ 15(3), 85-97. Recuperado de https://redie.uabc.mx/redie/article/view/562/818.

Villatte, A., Courtinat-Camps, A. and Léonardis, M. (2014). Typology of self-concept of adolescents in France: a comparison of gifted an nongifted French high school students. Roeper Review, 36(1), 30-42. Doi: doi/10.1080/02783193.2013.856828

Young, J., Young, J. and Ford, D. (2017). Standing in the Gaps: Examining the Effects of Early Gifted Education on Black Girl Achievement in STEM. Journal of Advance Academics, 28(4), 290-312. Doi: doi.org/10.1177/1932202X17730549 


\begin{tabular}{|c|c|}
\hline Rol de Contribución & Autor (es) \\
\hline Conceptualización & Alejandro Jesús Robles y Diana Irasema Cervantes Igual \\
\hline Metodología & Alejandro Jesús Robles \\
\hline Software & $\begin{array}{l}\text { Alejandro Jesús Robles Principal Diana Irasema Cervantes De } \\
\text { apoyo }\end{array}$ \\
\hline Validación & Alejandro Jesús Robles Diana Irasema Cervantes Igual \\
\hline Análisis Formal & Alejandro Jesús Robles y Diana Irasema Cervantes Igual \\
\hline Investigación & $\begin{array}{l}\text { Alejandro Jesús Robles principal Diana Irasema Cervantes de } \\
\text { apoyo }\end{array}$ \\
\hline Recursos & $\begin{array}{l}\text { Alejandro Jesús Robles Principal Diana Irasema Cervantes De } \\
\text { apoyo }\end{array}$ \\
\hline Curación de datos & $\begin{array}{l}\text { Alejandro Jesús Robles Principal Diana Irasema Cervantes De } \\
\text { apoyo }\end{array}$ \\
\hline $\begin{array}{l}\text { Escritura - Preparación } \\
\text { del borrador original }\end{array}$ & Alejandro Jesús Robles Diana Irasema Cervantes Igual \\
\hline Escritura - Revisión y edición & $\begin{array}{l}\text { Diana Irasema Cervantes Principal Alejandro Jesús Robles De } \\
\text { apoyo }\end{array}$ \\
\hline Visualización & Alejandro Jesús Robles Diana Irasema Cervantes Igual \\
\hline Supervisión & Alejandro Jesús Robles Diana Irasema Cervantes Igual \\
\hline Administración de Proyectos & Alejandro Jesús Robles Diana Irasema Cervantes Igual \\
\hline Adquisición de fondos & $\begin{array}{l}\text { Universidad Autónoma de Ciudad Juárez (apoyo con un aporte } \\
\text { económico) } \\
\text { Alejandro Jesús Robles Diana Irasema Cervantes Igual }\end{array}$ \\
\hline
\end{tabular}

